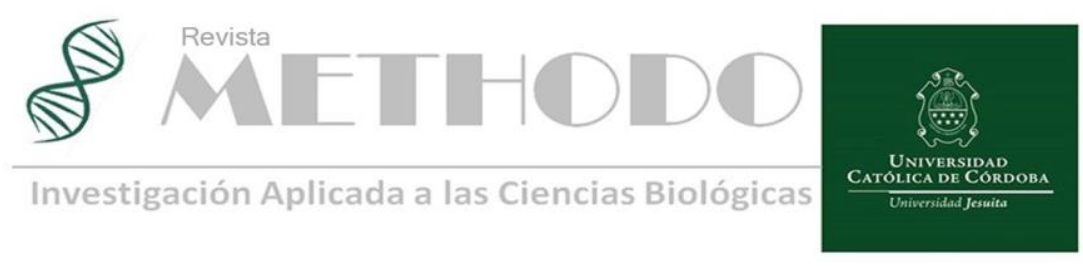

\title{
Oseointegración, aspectos que determinan su éxito. Revisión de la literatura.
}

\section{Osseointegration, aspects that determine its success. Literature review}

\author{
Ariel Damián Fochini ${ }^{1}$, Nicolás Leonardi ${ }^{2}$ \\ 1Universidad Católica de Córdoba. Facultad de Ciencias de la Salud. Carrera de Especialización en Implantología Oral \\ 2Universidad Católica de Córdoba. Facultad de Ciencias de la Salud, Cátedra Medicina Bucal, Escuela de Odontología. \\ Correspondencia Ariel Damián Fochini. Universidad Católica de Córdoba. Carrera de Especialización en Implantología Oral. Córdoba, Argentina. Email: \\ arielfochini@hotmail.com
}

\section{Resumen}

El éxito del implante dental depende en gran medida de las características químicas, físicas, mecánicas y topográficas de su superficie; ya que determinan la actividad de las células que se adhieren a la superficie del implante. Por lo tanto, la humectabilidad, geometría, topografía, rugosidad, energía superficial, nanoestructuras y el recubrimiento con materiales bioactivos tienen un impacto sustancial en el éxito y calidad del proceso de oseointegración.

Metodología: Se realizó una revisión de la literatura mediante la base de datos de MedLine con el motor de búsqueda Pubmed. Se seleccionaron, una vez aplicados los criterios de inclusión y exclusión, un total de 69 artículos.

Resultados: La combinación de estas propiedades determinan la calidad y grado de oseointegración. Valores moderados de hidrofilia $\left(30^{\circ}-60^{\circ}\right)$ y rugosidad $(\mathrm{Sa}=1-2 \mu \mathrm{m})$ favorecen la oseointegración, pero aún se desconocen los mecanismos biológicos subyacentes. La investigación actual sigue los enfoques biomiméticos: imitar la configuración 3D de la superficie ósea.

Palabras claves: Oseointegración, implante dental, superficie, diseño

\begin{abstract}
The success of a dental implant is highly dependent on its chemical, physical, mechanical, and topographic characteristics of its surface; because they determine the activity of the cell attachment that are close to the dental implant surface. Therefore, the chemical composition, wettability, geometry, topography, roughness, surface energy, nanostructures and the coating with bioactive materials have a substantial impact on the rate and quality ofthe osseointegration process.

Methodology: A literature review was performed using the MedLine database with the Pubmed search engine. A total of 69 articles were selected after applying the inclusion and exclusion criteria.

Results: The combination of these properties determine the quality and degree of osseointegration. Moderate values of hydrophilicity $\left(30^{\circ}-60^{\circ}\right)$ and roughness $(\mathrm{Sa}=1-2 \mu \mathrm{m})$ favor osseointegration but we still don't know the underlying biological mechanisms.

Current research follows biomimetic approaches: imitate the 3D configuration of the bone surface.
\end{abstract}

Revista Methodo: Investigación Aplicada a las Ciencias Biológicas. Universidad Católica de Córdoba. Jacinto Ríos 571 Bo Gral. Paz. X5004FXS. Córdoba. Argentina. Tel.: (54) 3514517299 / Correo: methodo@ucc.edu.ar / Web: methodo.ucc.edu.ar | ARTICULO DE REVISION Rev. Methodo 2020;5(4):156-164. 
Key words: Osseointegration, dental implant, surface, design.

\section{Introducción}

La implantología oral ha revolucionado la práctica de la odontología durante los últimos 15 años. Múltiples estudios han demostrado que la rehabilitación de los pacientes desdentados parciales y totales con implantes dentales es un procedimiento predecible ${ }^{1-3}$.

En este sentido, el pronóstico a largo plazo de la terapéutica con implantes está relacionado con el desarrollo del fenómeno de la oseointegración y de una favorable respuesta de los tejidos mucoperiimplantarios ${ }^{4-5}$. El material más frecuentemente utilizado en la realización de los implantes orales ha sido el titanio comercialmente puro, debido a que presenta una gran biocompatibilidad y constituye el material ideal para conseguir la oseointegración con éxito a largo plazo tras la carga funcional ${ }^{6-7}$.

En la década de los años 60, Branemark y colaboradores $^{8-9}$, introdujeron el término oseointegración para referirse a la aceptación y anclaje de piezas de titanio colocadas en el hueso maxilar. La oseointegración es la conexión firme, estable y duradera entre un implante sujeto a carga y el hueso que lo rodea.

El éxito de esta conexión o interfase huesoimplante depende de factores biológicos y sistémicos del paciente y de las características del implante y su superficie ${ }^{10-12}$.

Por su parte, la interfase hueso-implante dental se caracteriza por las propiedades favorables al crecimiento y formación de nuevo hueso alveolar que posee el implante en su superficie y por el diseño del mismo, lo que le permite distribuir adecuadamente las cargas mecánicas ejercidas durante la masticación ${ }^{13-17}$. Por lo tanto, esta interfase debe considerarse como el resultado de la interacción de un conjunto de factores que modulan la respuesta biológica y que determinan el éxito de la oseointegración, entre los que se encuentran la respuesta inmune del paciente, el procedimiento de inserción, las características fisiológicas del hueso receptor, los factores mecánicos del implante, su superficie y la acción de fuerzas mecánicas sobre el hueso y el implante ${ }^{18-21}$

\section{Objetivos}

1- Analizar la literatura sobre las propiedades bioactivas de la superficie que influyen en la capacidad de oseointegración.
2- Revisar en la bibliografía respecto a los tratamientos superficiales disponibles para la fabricación de implantes y determinar qué propiedades aporta cada uno.

3- Determinar la influencia biológica y biomecánica del diseño superficial del implante de titanio y reflejar en la actualidad hacia dónde apuntan las futuras investigaciones.

\section{Interfase hueso-implante}

El hueso alveolar está formado por dos estructuras: el proceso alveolar y la cortical alveolar.

Una lesión en este tipo de hueso, como la producida durante el procedimiento de inserción de un implante dental, se recupera siguiendo las etapas del proceso de cicatrización del hueso intramembranoso ${ }^{21-26}$.

Este proceso consiste de cuatro etapas, cada una asociada a un evento biológico característico (Figura 1): 1) formación del hematoma (sangrado y coagulación), 2) degradación del coagulo y limpieza de la herida (fibrinólisis), 3) formación de tejido granular (fibroplasia y angiogénesis), y 4) síntesis y mineralización de nuevo hueso (modelamiento y remodelamiento óseo) ${ }^{26-27}$

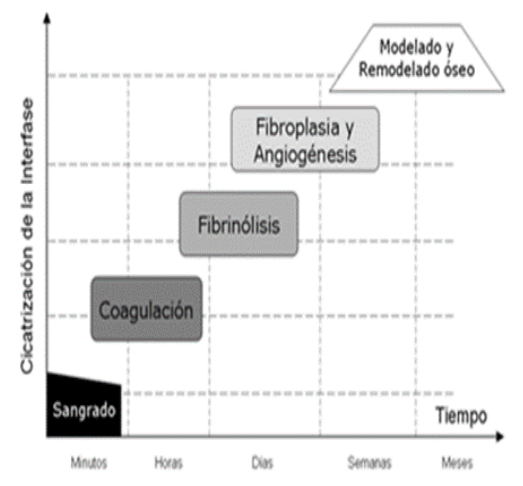

Figura 1 - Etapas biológicas que sigue la cicatrización de la interfase hueso-implante.

Las características de la lesión causada en el hueso alveolar como consecuencia del procedimiento quirúrgico de inserción del implante dental determinan la viabilidad del proceso de cicatrización y del nuevo hueso ${ }^{28}$. Estas características están en relación directa con la calidad del hueso y con el procedimiento quirúrgico utilizado (Figura 2). Se denomina calidad de hueso a la relación de cantidad existente entre la proporción de hueso cortical de la cortical 
alveolar y la proporción de hueso trabecular del proceso alveolar ${ }^{29-30}$.

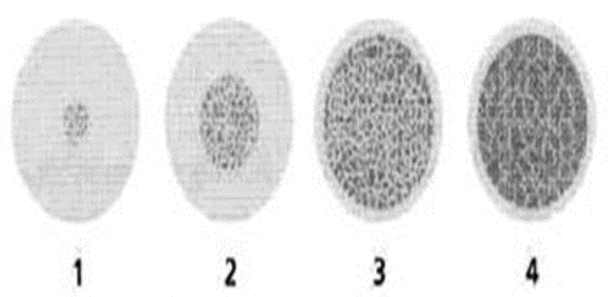

Figura 2. diferentes tipos y calidad de hueso

La calidad de hueso es importante en implantología dental debido a que representa un indicador de viabilidad para un tratamiento $\mathrm{y}$ procedimiento de inserción determinado. Esta dualidad entre estabilidad y tiempo de cicatrización ha permitido la aparición de nuevas técnicas de fabricación de implantes dentales y nuevos protocolos de inserción que mejoran la tasa y velocidad de cicatrización independiente de la calidad de hueso, con lo cual se impulsa el diseño de implantes oseointegrables destinados a ser utilizados en zonas con hueso mayoritariamente trabecular ${ }^{31-34}$.

\section{El implante dental y su diseño macro y microscópico}

Un implante dental es un dispositivo hecho de un material biológicamente inerte que es insertado mediante cirugía en el hueso alveolar y que sustituye la raíz de un diente ausente ${ }^{35}$.

El macro diseño de los implantes está destinado a mejorar tres aspectos clínico-biológicos, el aumento de la estabilidad primaria, la adaptación a los defectos anatómicos y a los alveolos postextraccion, y el mantenimiento de la cresta ósea alveolar marginal a largo plazo ${ }^{36}$.

El concepto radicular de los implantes intraóseos representa la forma más utilizada, actualmente, como un tornillo cilíndrico o en forma de raíz dental en la que se pueden diferenciar tres partes (cuerpo, cabeza y porción transmucosa) ${ }^{37-42}$.

El cuerpo es la parte fundamental del implante desde un punto de vista biológico, ya que, colocado quirúrgicamente en el interior del hueso, permite su oseointegración. Dependiendo de la morfología y el procedimiento quirúrgico utilizado para conseguir anclaje primario ${ }^{35-39}$.

Los implantes roscados presentan espiras propias de un tornillo y su colocación endoósea se realiza labrando un lecho mediante un macho de tarraja que permitirá el posterior roscado del implante en conjunto con el uso de fresas ad hoc, salvo en implantes muy delgados. Requiere más pasos quirúrgicos, pero presenta una buena fijación primaria. En estos implantes el elemento diferencia es el perfil de sus roscas. Las roscas pueden tener, según su perfil, forma de "V", de cuadrado o asimétricas en la porción superior de la rosca más larga que en la inferior (árbol de navidad). También existen diseños que varían el perfil y la densidad de las roscas a lo largo de la superficie externa del implante con el fin de que la fijación no sea igual de agresiva en toda su longitud $^{40-42}$.

Actualmente los implantes roscados presentan un diseño que no necesitan labrado de un lecho y además proporcionan un alto grado de estabilidad primaria. Estos implantes denominados autorroscables solo se utilizan en ciertos casos particulares donde se necesitan diámetros reducidos $^{35-42}$.

La superficie de los implantes o micro superficie es, en la actualidad, un objetivo preferente de los investigadores y de la industria, debido a que mediante la variación en su morfología y propiedades podemos influir sobre la intensidad y velocidad del proceso de oseointegración ${ }^{43-44}$.

La calidad de la superficie del implante dependerá de sus características físicas, químicas y topográficas. La composición química, las impurezas de la superficie, así como el grosor y la estructura de la capa superficial influyen en el grado de biocompatibilidad ${ }^{45}$.

Por lo tanto, en función de la superficie podemos mejorar tres aspectos como son la aposición ósea acelerada en la fase inicial de la cicatrización, el aumento en el área de la superficie de contacto del implante con el hueso y la mejora del anclaje del implante ${ }^{45-46}$.

También existen otras propiedades físicas que influyen a corto y largo plazo en la oseointegración como la energía superficial y la carga superficial. La energía superficial viene definida por la densidad de la carga y la polaridad, es decir que una superficie con energía alta tiene gran afinidad por la absorción, influyendo sobre las proteínas para formar un recubrimiento primario ventajoso y así mejorar la oseointegración del implante. La carga superficial es la habilidad para absorber iones positivos o negativos en superficie, siendo este un factor fundamental para la nucleación de otros materiales sobre la superficie del implante ${ }^{47-}$ 49 .

\section{Métodos de tratamiento de la superficie de los implantes}

Existen diversos métodos para tratar la superficie del implante y convertirla en rugosa con el fin de incrementar el grado de oseointegración al 
aumentar la adhesión a las proteínas que influyen en la adherencia celular ${ }^{43-49}$.

\section{Recubrimiento con técnicas de adición}

Técnicas de spray de plasma de partículas de hidroxiapatita o de titanio. Puede realizarse la deposición química o física de vapor en la que se recubre el implante de una capa de diamante; el rociado de fosfato cálcico por radiofrecuencia; el aumento de la capa de óxido de titanio por oxidación anódica ${ }^{43-51}$.

\section{Tratamiento con técnicas de sustracción}

Arenado con partículas (cristal, óxido de titanio, óxido de aluminio, óxido de zirconio) de diversos tamaños. Esta técnica consiste en la proyección de diversas partículas de elevada pureza a gran velocidad y presión sobre la superficie del implante. Este proceso mejora las cualidades mecánicas, la resistencia a la fatiga y la resistencia a la corrosión ${ }^{52}$.

Grabado ácido o doble grabado (clorhídrico, sulfúrico, fluorhídrico, nítrico). Debe hacerse por inmersión en ácidos no muy concentrados, durante poco tiempo y a una temperatura controlada. Este método aumenta la capacidad de oseointegración, la superficie de contacto y la estabilidad primaria del implante ${ }^{52}$.

Arenado y grabado ácido. Esta técnica combina las dos anteriores, se realiza un arenado y posteriormente un grabado ácido. De esta forma se pretende incrementar la velocidad y el grado de oseointegración ${ }^{52}$.

\section{Modificación de la superficie del material}

Esta técnica de tratamiento de la superficie de los implantes no añade ni sustrae material, sino que modifica la superficie por tratamientos físicos, con rayo electro-térmico; con láser; o mediante una implantación iónica (electrones que se aceleran a elevadas velocidades y se hacen incidir con la superficie a tratar) ${ }^{53}$.

Una superficie es dinámicamente biocompatible cuando sus propiedades y las condiciones biofuncionales del complejo anatómico en el que está insertada permanecen estables con el paso del tiempo, respetando las funciones del órgano. Todas las superficies actuales deben cumplir esta premisa $^{54}$.

A pesar de que la biocompatibilidad y la velocidad de oseointegración de las superficies recubiertas de hidroxiapatita están demostradas, también se han aportado referencias en cuanto a la variabilidad de la fuerza de unión entre el cuerpo de titanio del implante, el recubrimiento y su distribución poco uniforme. Cuanto mayor es el porcentaje de cristalinidad y menos es la parte amorfa de la hidroxiapatita que recubre el implante mayor calidad tiene, siendo su reabsorción menor y más lenta ${ }^{53-57}$.

En función de la rugosidad media en superficie, las micro superficies de los implantes se clasifican en pulidas (entre 0 y $0,4 \mu \mathrm{m}$ ); con rugosidad mínima o maquinada (entre 0;5 y $1 \mu \mathrm{m}$ ); con rugosidad moderada (entre 1 y $2 \mu \mathrm{m}$ ) y con rugosidad elevada (más de $2 \mu \mathrm{m}$, como son las superficies con partículas de titanio o hidroxiapatita) ${ }^{35-42-44}$.

Entre las superficies de rugosidad moderada se encuentran Tiobast de Astra Tech ${ }^{\circledR}$, Frialit de Friadent $₫$ y RBM de Biohorizons $₫$ por arenado de partículas de óxido de titanio, óxido de aluminio y fosfato cálcico; las de arenado y grabado como SLA de Straumann (B; las obtenidas por doble grabado acido como son Osseotite de Biomet 3I ® y por oxidación anódica como TiUnite de Nobel Biocare ${ }^{\circledR 58}$.

Recientemente, las novedades en las superficies están orientadas a proporcionar bioactividad. Por lo tanto, la investigación en este campo tiende a añadir a las superficies con rugosidad determinada de los implantes modificaciones para acelerar el proceso de oseointegración a su alrededor, por lo que esta modificación de la superficie original presenta una mayor energía libre superficial y una hidrofilia superior, con unos ángulos de contacto con el agua de cero grados, en comparación con los 139,9 grados de la superficie original. SLA Active pretende mantener la hidrofilia y la elevada energía superficial de dióxido de titanio hasta la inserción quirúrgica del implante ${ }^{58}$.

\section{Oseointegración}

Como se mencionó anteriormente la oseointegración es la aceptación y adaptación funcional de un implante insertado en el hueso. El éxito depende de la función de dos procesos previos: la osteoinducción y la osteoconducción ${ }^{15}$. La osteoinducción es el proceso a través del cual las células madre se diferencian en las células osteogénicas que forman el tejido óseo. La deposición de nuevo hueso por parte de estas células se conoce como osteogénesis. Existen dos tipos de osteogénesis: osteogénesis a distancia y osteogénesis de contacto. En la primera, el tejido óseo se forma desde la superficie del hueso circundante. En la segunda, la formación de tejido óseo se produce desde la superficie del implante. Es decir, la formación de hueso en la región periprotésica tiene dos direcciones: desde la superficie del hueso circundante hacia el implante

Revista Methodo: Investigación Aplicada a las Ciencias Biológicas. Universidad Católica de Córdoba. Jacinto 
desde la superficie del implante hacia el hueso circundante ${ }^{15-22}$.

Sin embargo, y a pesar que la osteogénesis de contacto forma tejido óseo a una velocidad $30 \%$ mayor que la osteogénesis a distancia ${ }^{22}$, la formación desde la superficie del implante implica que dicha superficie permita su colonización por parte de células de origen mesenquimal ${ }^{21}$. Esta colonización, denominada osteoconducción, corresponde a la formación de hueso sobre una superficie de material bioactivo ${ }^{15-21}$. Este fenómeno depende esencialmente de la biocompatibilidad del material y de sus características superficiales. Como consecuencia de la colonización de la superficie del implante por parte de las células osteoprogenitoras, se forma una interfase de contacto entre el implante y los tejidos que lo rodean ${ }^{45-58}$.

Este contacto puede ser de dos tipos: el contacto con el hueso o tejido duro, y el contacto con el tejido fibroso o blando. Se ha reportado que el contacto directo entre el hueso vivo y la superficie del implante forma una fuerte matriz extracelular de unión tanto estructural como funcional que incrementa con el tiempo, promueve la osteogénesis reparativa en la interfase y permite la fijación del implante gracias a su mineralización. En el caso de la interfase con el tejido blando, se ha reportado que las células epiteliales forman un fuerte collar alrededor del implante que carece de signos de respuesta inflamatoria y que crea una fina capa de tejido conectivo poco vascularizada en las cercanías de la superficie del implante a partir de la cual comienza la regeneración tisular ${ }^{59-}$ 62

Estos contactos entre el tejido biológico y el implante están relacionados con las características topográficas en la superficie del implante. Existen tres escalas de topografía superficial, cada una con ventajas respecto al comportamiento del implante y la formación de la interfase hueso-implante (Figura 3).

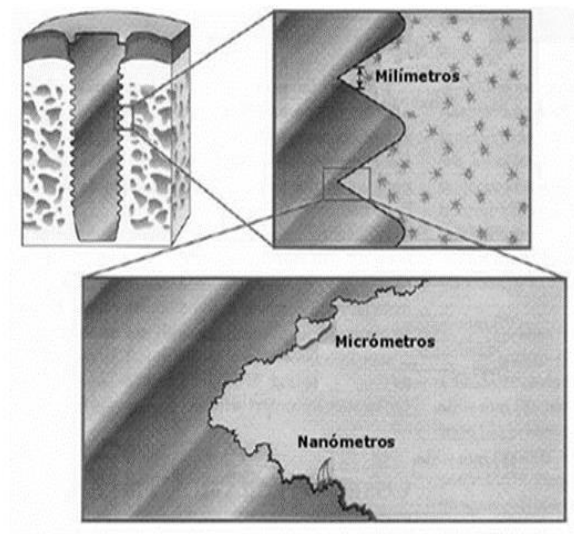

Figura 3. Las tres escalas de modificaciones en la superficie de un implante.
Se ha establecido que las modificaciones superficiales en las dimensiones menores a un micrómetro tienen influencia en la morfología, orientación y adhesión celular, mientras que las dimensiones entre uno y cien micrómetros están asociadas con la formación de hueso, y las dimensiones superiores a los cien micrómetros, especialmente la topografía roscada, están relacionadas con el soporte y estabilidad del implante frente a la acción mecánica ${ }^{63-65}$.

Mientras la macrotopografía, es decir, el diseño acanalado del cuerpo del implante está relacionado con la resistencia a esfuerzos y la estabilidad inicial, la microtopografía, es decir, el tratamiento superficial, proporciona a la superficie del implante un patrón similar al dejado por el frente de resorción de osteoclastos en la superficie del hueso durante el remodelamiento ${ }^{66}$. Este patrón superficial permite que la línea de cementación secretada por los precursores osteoblásticos se entrelace con la superficie del implante y asegure la formación del nuevo hueso. De la misma manera, el recubrimiento de la superficie del implante puede incrementar la absorción de integrinas. Las integrinas son cadenas de proteínas responsables de la adhesión a nivel celular entre el citoesqueleto de la célula y un sustrato, en este caso, la superficie del implante. Esta unión se logra mediante la combinación de secuencias proteicas específicas que se encuentran tanto en el citoesqueleto de la célula como en el sustrato. La unión de estas secuencias activa vías de señalización que modifican el comportamiento de la célula, haciendo por ejemplo que prolifere o se diferencie ${ }^{50-67-68 .}$

El éxito del tratamiento con implantes dentales se produce cuando se consigue su oseointegración en el sentido del concepto introducido por Branemark $^{67}$. Deben cumplirse una serie de requisitos para lograr la oseointegración, aunque actualmente están sujetos a revisión respecto a que fueron preconizados inicialmente, especialmente en lo que se refiere a la puesta en función del implante ${ }^{8-9-68-69}$.

\section{Conclusión}

La humectabilidad y principalmente la química y topografía superficial determinan la calidad y grado de oseointegración. La combinación de estas propiedades actúa sinérgicamente en la promoción de la oseointegración.

En la actualidad las técnicas de fabricación más usadas son: recubrimiento (presenta limitaciones), grabado ácido (preferible dual), arenado y SLA (granallado + grabado ácido). Todas las técnicas mejoran la oseointegración por aumento de la rugosidad, 
algunas además estimulan la interacción química de osteoblastos con sustancias depositadas en la superficie.

El manejo de las características superficiales del implante es una herramienta útil para el odontólogo, pero aún desconocemos los mecanismos biológicos subyacentes. Valores moderados de hidrofilia (ángulos de contacto $30^{\circ}$ $60 \circ)$ y de rugosidad $(\mathrm{Sa}=1-2 \mu \mathrm{m})$ favorecen la oseointegración y aumento del BIC (Bone Implant Contact), incluso en zonas regeneradas.

Para avanzar en este campo, es necesario intensificar la colaboración interdisciplinaria entre medicina e ingeniería. La investigación actual sigue los enfoques biomiméticos, imitar la configuración 3D de la superficie ósea tras la resorción ya que promueve la osteointegración y el anclaje mecánico del implante.

\section{Bibliografía}

1. Buser D, Mericske-Stern RD, Bernard JP. Long-term evaluation of non-submerged ITI implants. Part 1: 8-year life table analysis of a prospective multi-center study with 2359 implants. Clin Oral Impl Res 1997; 8: 161-72.

2. Albrektsson, T.; Chrcanovic, B.; Molne, J.; Wennerberg, A. Foreign body reactions, marginal bone loss and allergies in relation to titanium implants. Eur. J. Oral Implantol.2018, 11, S37-S46.

3. Coelho PG, Jimbo R, Tovar N, Bonfante EA. Osseointegration: hierarchicaldesigning encompassing the macrometer, micrometer, and nanometer length scales.Dent Mater. 2015 Jan;31(1):37-52.

4. Salvi GE, Bosshardt DD, Lang NP, Abrahamsson I, et al. Temporal sequence of hardand soft tissue healing around titanium dental implants. Periodontol 2000. 2015Jun;68(1):135-52.

5. Meng H-W, Chien EY, Chien H-H. Dental implant bioactive surface modifications and their effects on osseointegration: a review. Biomark Res. 2016;4(Dec 14):24.

6. Ogle OE. Implant surface material, design, and osseointegration. Dent Clin NorthAm. 2015 Apr;59(2):505-20.

7. Albertini M, Fernandez-Yague M, Lázaro P, Herrero-Climent $M$, et al. Advances in surfaces and osseointegration in implantology. Biomimetic surfaces. Med Oral Patol Oral Cir Bucal. 2015 May 1;20(3):31625.
8. Adell R, Lekholm U, Rockler B, Branemark PI. 15-year study of osseointegrated implants in the treatment of the edentulous jaw. Int. J. Oral Surg. 1981; 10: 387-416.

9. Branemark R, Branemark PI, Rydevik B, Myers R. Osseointegration in skeletal reconstruction and rehabilitation. A review. J. Rehab. Reseach Dev. 2001; 38 (2): 175-181.

10. Feller L, Jadwat Y, Khammissa RAG, Meyerov R, et al. Cellular responses evokedby different surface characteristics of intraosseous titanium implants. Biomed Res Int.2015; 2015:171945.

11. Annunziata M, Guida L. The Effect of Titanium Surface Modifications on DentalImplant Osseointegration. Front Oral Biol. 2015; 17:62-77.

12. Velasco-Ortega E, Alfonso-Rodríguez CA, Monsalve-Guil L, España-López JiménezGuerra A, et al. Relevant aspects in the surface properties in titanium dentalimplants for the cellular viability. Mater Sci Eng C Mater Biol Appl. 2016 Jul 1;64(Jul1):1-10.

13. Liu P, Hao Y, Zhao Y, Yuan Z, et al. Surface modification of titanium substrates for enhanced osteogenetic and antibacterial properties. Colloids Surf B Biointerfaces. 2017 Dec 1;160(Dec 1):110-6

14. Andrukhov O, Huber R, Shi B, Berner S, Rausch-Fan X, et al. Proliferation, behavior, and differentiation of osteoblasts on surfaces of different microroughness. Dent Mater. 2016 Nov;32(11):1374-84.

15. Sul Y-T, Kang B-S, Johansson C, Um H-S, et al. The roles of surface chemistry and topography in the strength and rate of osseointegration of titanium implants in bone. J Biomed Mater Res A. 2009 Jun 15;89(4):942-50.

16. Schmid J, Brunold S, Bertl M, Ulmer H, Kuhn $\mathrm{V}$, et al. Biofunctionalization of onplants to enhance their osseointegration. Int J Stomatol Occlusion Med. 2014 Dec 5;7(4):105-10.

17. Bressan E, Sbricoli L, Guazzo R, Tocco I, et al. Nanostructured surfaces of dental implants. Int J Mol Sci. 2013 Jan 17;14(1):1918-31.

18. Shibata Y, Tanimoto Y, Maruyama N, Nagakura M. A review of improved fixation methods for dental implants. Part II: biomechanical integrity at bone-implant interface. J Prosthodont Res. 2015 Apr;59(2):84-95. 
19. von Wilmowsky C, Moest T, Nkenke E, Stelzle F, et al. Implants in bone: part I. A current overview about tissue response, surface modifications and future perspectives. Oral Maxillofac Surg. 2014 Sep;18(3):24357.

20. Olivares-Navarrete R, Hyzy SL, Berg ME, Schneider JM, et al. Osteoblast lineage cells can discriminate microscale topographic features on titanium-aluminum-vanadium surfaces. Ann Biomed Eng. 2014 Dec;42(12):2551-61.

21. Smeets R, Stadlinger B, Schwarz F, BeckBroichsitter B, et al. Impact of Dental Implant Surface Modifications on Osseointegration. Biomed Res Int. 2016; 2016:6285620.

22. Dohan Ehrenfest DM, Coelho PG, Kang B-S, Sul Y-T, et al. Classification of osseointegrated implant surfaces: materials, chemistry and topography. Trends Biotechnol. 2010 Apr;28(4):198-206.

23. Buser, D.; Janner, S.F.; Wittneben, J.G.; Bragger, U.; et al. 10-year survival and success rates of 511 titanium implants with a sandblasted and acid-etched surface: A retrospective study in 303partially edentulous patients. Clin. Implant Dent. Relat. Res. 2012; 14: 839-851

24. Rupp F, Liang L, Geis-Gerstorfer J, Scheideler L, et al. Surface characteristics of dental implants: A review. Dent Mater. 2018 Jan;34(1):40-57.

25. Shibata Y, Tanimoto Y. A review of improved fixation methods for dental implants. Part I: Surface optimization for rapid osseointegration. J Prosthodont Res. 2015 Jan;59(1):20-33.

26. Olivares-Navarrete R, Rodil SE, Hyzy SL, Dunn GR, et al. Role of integrin subunits in mesenchymal stem cell differentiation and osteoblast maturation on graphitic carboncoated microstructured surfaces. Biomaterials. 2015 May; 51:69-79.

27. Hotchkiss KM, Reddy GB, Hyzy SL, Schwartz Z, et al. Titanium surface characteristics, including topography and wettability, alter macrophage activation. Acta Biomater. 2016 Feb;31(Feb):425-34.

28. Gittens RA, Scheideler L, Rupp F, Hyzy SL, et al. A review on the wettability of dental implant surfaces II: Biological and clinical aspects. Acta Biomater. 2014 Jul;10(7):290718.
29. Livne S, Marku-Cohen S, Harel N, Piek D, et al. [The influence of dental implant surface on osseointegration: review]. Refuat Hapeh Vehashinayim. 2012 Jan;29(1):41-6, 66.

30. Rupp F, Gittens RA, Scheideler L, Marmur A, et al. A review on the wettability of dental implant surfaces I: theoretical and experimental aspects. Acta Biomater. 2014 Jul;10(7):2894-906.

31. Pegueroles M, Aparicio C, Bosio M, Engel E, et al. Spatialorganization of osteoblast fibronectin matrix on titanium surfaces: effects of roughness, chemical heterogeneity and surface energy. Acta Biomater. 2010 Jan;6(1):291-301.

32. Gittens RA, Olivares-Navarrete R, Cheng A, Anderson DM, et al. The roles of titanium surface micro/nanotopography and wettability on the differential response of human osteoblast lineage cells. Acta Biomater. 2013 Apr;9(4):6268-77.

33. Chan KH, Zhuo S, Ni M. Priming the Surface of Orthopedic Implants for Osteoblast Attachment in Bone Tissue Engineering. Int $\mathbf{J}$ Med Sci. 2015;12(9):701-7.

34. Gittens RA, Olivares-Navarrete R, Schwartz $\mathrm{Z}$, Boyan BD. Implant osseointegration and the role of microroughness and nanostructures: lessons for spine implants. Acta Biomater. 2014 Aug;10(8):3363-71

35. Jemat A, Ghazali MJ, Razali M, Otsuka Y. Surface Modifications and Their Effects on Titanium Dental Implants. Biomed Res Int. 2015; 2015:791725.

36. Barfeie A, Wilson J, Rees J. Implant surface characteristics and their effect on osseointegration. $\mathrm{Br}$ Dent J. 2015 Mar $13 ; 218(5)$

37. Tobin EJ. Recent coating developments for combination devices in orthopedic and dental applications: A literature review. Adv Drug Deliv Rev. 2017 Mar;112(Mar):88- 100.

38. Compton JT, Lee FY. A review of osteocyte function and the emerging importance of sclerostin. J Bone Joint Surg Am. 2014 Oct 1;96(19):1659-68.

39. Virdi AS, Irish J, Sena K, Liu M, et al. Sclerostin antibody treatment improves implant fixation in a model of severe osteoporosis. J Bone Joint Surg Am. 2015 Jan 21;97(2):133-40.

40. Stadlinger B, Korn P, Tödtmann N, Eckelt U, et al. Osseointegration of biochemically 
modified implants in an osteoporosis rodent model. Eur Cell Mater. 2013 Jul 8; 25:326-4040.

41. Ferraris S, Spriano S. Antibacterial titanium surfaces for medical implants. Mater Sci Eng C Mater Biol Appl. 2016 Apr 1;61(1):965-78.

42. Rivera-Chacon DM, Alvarado-Velez M, Acevedo-Morantes CY, Singh SP, et al. Fibronectin and vitronectin promote human fetal osteoblast cell attachment and proliferation on nanoporous titanium surfaces. J Biomed Nanotechnol. 2013 Jun;9(6):10927.

43. Trindade R, Albrektsson T, Galli S, Prgomet $\mathrm{Z}$, et al. Osseointegration and foreign body reaction: titanium implants activate the immune system and suppress bone resorption during the first 4 weeks after implantation. Clin Implant Dent Relat Res. 2018; 20:82-91

44. Albrektsson T, Chrcanovic B, Jacobsson M, Wennerberg A. Osseointegration of implants - a biological and clinical overview. JSM Dent Surg. 2017; 2:1-6.

45. Van Velzen, F.J.; Ofec, R.; Schulten, E.A.; Ten Bruggenkate, C.M. 10-year survival rate and the incidence of peri-implant disease of 374 titanium dental implants with a SLA surface: A prospective cohort study in 177 fully and partially edentulous patients. Clin. Oral Implants Res. 2015; 26: 1121-1128.

46. Wheelis SE, Montaño-Figueroa AG, Quevedo-Lopez M, Rodrigues DC. Effects of titanium oxide surface properties on boneforming and soft tissue-forming cells. Clin Implant Dent Relat Res. 2018;20(5): 838-847.

47. Ramaglia L, Postiglione L, Di Spigna G, Capece G, et al. Sandblastedacid- etched titanium surface influences in vitro the biological behavior of SaOS-2 human osteoblast-like cells. Dent Mater J. 2011;30(2):183-92.

48. Albrektsson, T., \& Wennerberg, A. On osseointegration in relation to implant surfaces. Clinical Implant Dentistry and Related Research. 2019; 21(1):4-7

49. Al Mustafa M, Agis H, Müller HD, Watzek G, et al. In vitro adhesion of fibroblastic cells to titanium alloy discs treated with sodium hydroxide. Clin Oral Implants Res. 2015;26(1):15-19.

50. Yamamura K, Miura T, Kou I, Muramatsu T, et al. Influence of various superhydrophilic treatments of titanium on the initial attachment, proliferation, and differentiation of osteo-blast-like cells. Dent Mater J. 2015;34(1):120-127.

51. Lang NP, Salvi GE, Huynh-Ba G, Ivanovski $\mathrm{S}$, et al. Early osseointegration to hydrophilic and hydrophobic implant surfaces in humans. Clin Oral Implants Res. 2011; 22:349-356.

52. Yeo, I.S.; Min, S.K.; Kang, H.K.; Kwon, T.K, et al. Identification of a bioactive core sequence from human laminin and its applicability to tissue engineering. Biomaterials 2015; 73: 96-109.

53. Pjetursson BE, Thoma D, Jung R, Zwahlen M, et al. A systematic review of the survival and complication rates of implant-supported fixed dental prostheses (FDPs) after a mean observation period of at least 5 years. Clin Oral Implants Res. 2012 Oct;23 Suppl 6:2238.

54. Wennerberg A, Albrektsson T. Effects of titanium surface topography on bone integration: a systematic review. Clin Oral Implants Res. 2009 Sep;20 Suppl 4(Sept):172-84.

55. Velasco E, Jos A, Pato J, Camean A, et al. In vitro evaluation of citotoxicity and genotoxixity of comercial titanium alloy for dental implantología. Mutation Res 2010; 702:17-23

56. Oztel, M.; Bilski, W.M.; Bilski, A. Risk factors associated with dental implant failure: A study of 302 implants placed in a regional center. J. Contemp. Dent. Pract. 2017: 18: 705-709.

57. Asensio, G.; Vazquez-Lasa, B.; Rojo, L. Achievements in the Topographic Design of Commercial Titanium Dental Implants: Towards Anti-Peri-Implantitis Surfaces. J. Clin. Med. 2019; 8: 1982.

58. Nicolau, P.; Guerra, F.; Reis, R.; Krafft, T, et al. 10-year outcomes with immediate and early loaded implants with a chemically modified SLA surface. Quintessence Int. 2018; 50: 2-12.

59. Ahn, T.K.; Lee, D.H.; Kim, T.S.; Jang, G.C, et al. Modification of Titanium Implant and Titanium Dioxide for Bone Tissue Engineering. Adv. Exp. Med. Biol. 2018; 1077: 355-368.

60. Souza, J.C.M.; Sordi, M.B.; Kanazawa, M.; Ravindran, $\mathrm{S}$, et al. Nano-scale modification of titanium implant surfaces to enhance osseointegration. Acta Biomater. 2019; 94: 112-131. 
61. Joos U, Wiesmann HP, Szuwart T, Meyer U. Mineralization at the interface of implants. Int. J. Oral Maxillofac. Surg. 2006; 35: 783790.

62. Abuhussein H, Pagni G, Rebaudi A, Wang HL. The effect of thread pattern upon implant osseointegration. Clin Oral Implants Res. 2010 Feb;21(2):129-36.

63. Kim, S.; Choi, J.Y.; Jung, S.Y.; Kang, H.K, et al. A laminin-derived functional peptide, PPFEGCIWN, promotes bone formation on sandblasted, large-grit, acid-etched titanium implant surfaces. Int. J. Oral Maxillofac. Implants. 2019, 34, 836-844

64. Albrektsson $\mathrm{T}$, Berglundh $\mathrm{T}$, Lindhe $\mathrm{J}$. Osseointegration: Historic background and current concepts. En: Lindhe J, Karring T, Lang N, eds. Clinical Periodontology and Implant Dentistry. Blackwell Munksgaard, 2003: 809-820.

65. Albrektsson T, Chrcanovic B, Jacobsson M, Wennerberg A. Osseointegration of implants - a biological and clinical overview. JSM Dent Surg. 2017; 2:1-6.

66. Le Guéhennec L, Soueidan A, Layrolle P, Amouriq Y. Surface treatments of titanium dental implants for rapid osseointegration. Dent Mater. 2007 Jul;23(7):844-54.

67. Avila G, Misch K, Galindo-Moreno P, Wang H-L. Implant surface treatment using biomimetic agents. Implant Dent. 2009 Feb;18(1):17-26.

68. Thakral G, Thakral R, Sharma N, Seth J, et al. Nanosurface - the future of implants. J Clin Diagn Res. 2014 May;8(5): ZE07-10.

69. Ogawa T. Ultraviolet photofunctionalization of titanium implants. Int J Oral Maxillofac Implants. 2014;29(1): e95-e102.

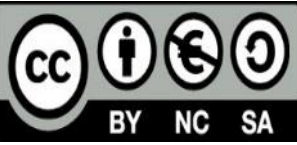

